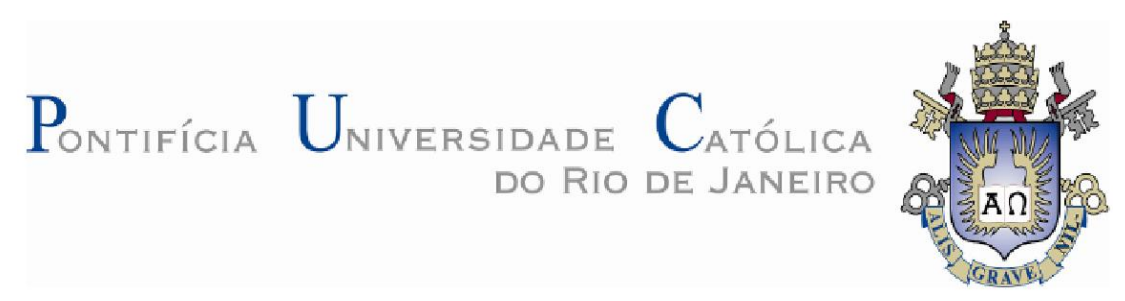

Danilo Andrés Jiménez Ugalde

Caracterização e propriedades mecânicas de conglomerados

Dissertação de Mestrado

Dissertação apresentada ao Programa de PósGraduação em Engenharia Civil da PUC-Rio como requisito parcial para obtenção do título de Mestre em Engenharia Civil.

Orientador: Prof. Sergio Augusto Barreto da Fontoura

Rio de Janeiro

Dezembro de 2015 


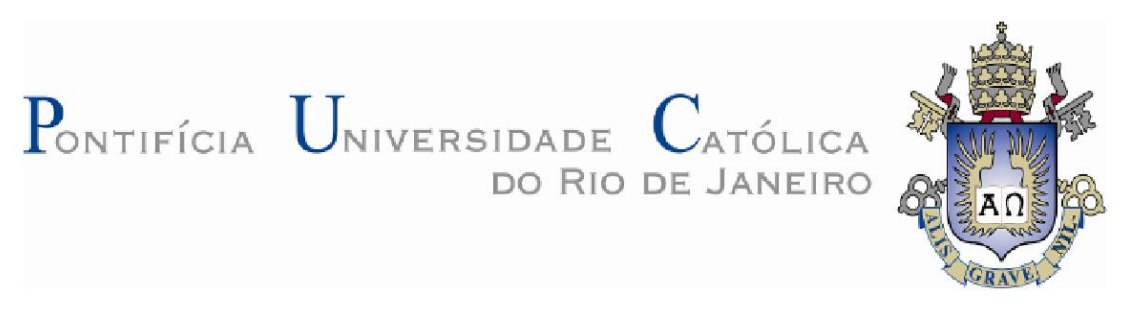

Danilo Andrés Jiménez Ugalde

\section{Caracterização e propriedades mecânicas de conglomerados}

Dissertação apresentada como requisito parcial para obtenção do grau de Mestre pelo Programa de PósGraduação em Engenharia Civil da PUC-Rio. Aprovada pela Comissão Examinadora abaixo assinada.

Prof. Sergio Augusto Barreto da Fontoura Orientador Departamento de Engenharia Civil - PUC-Rio

Prof. Alberto de Sampaio Ferraz Jardim Sayão

Departamento de Engenharia Civil - PUC-Rio

Prof. Milton Assis Kanji Consultor Independente - USP

Prof. Márcio da Silveira Carvalho Coordenador Setorial do Centro Técnico Científico - PUC-Rio 
Todos os direitos reservados. É proibida a reprodução total ou parcial do trabalho sem autorização da universidade, do autor e do orientador.

\section{Danilo Andrés Jiménez Ugalde}

Graduou-se em Engenharia Civil pela Universidade de Costa Rica - UCR (Costa Rica) em 2007. Principais áreas de interesse: mecânica das rochas, estabilidade de taludes, excavações subterrâneas, geomecânica computacional e engenharia de barragens.

Ficha Catalográfica

Jiménez Ugalde, Danilo Andrés

Caracterização e propriedades mecânicas de conglomerados / Danilo Andrés Jiménez Ugalde; orientador: Sergio Augusto Barreto da Fontoura 2015.

139 f. : il.(color.); $30 \mathrm{~cm}$

Dissertação

(mestrado)-Pontifícia

Universidade Católica do Rio de Janeiro,

Departamento de Engenharia Civil, 2015.

Inclui bibliografia

1. Engenharia civil - Teses. 2. Caracterização de conglomerados. 3. Ensaios de laboratório. 4. Método dos elementos finitos. 5. Bimrocks. I. Fountoura, Sergio A.B. da. II. Pontifícia Universidade Católica do Rio de Janeiro. Departamento de Engenharia Civil. III. Título. 


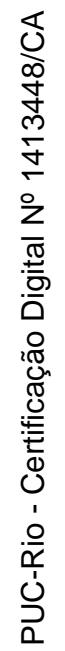

À Minha Família 


\section{Agradecimentos}

À Faperj e à Capes, pelos auxílios concedidos, sem os quais este trabalho não poderia ter sido realizado.

À Anita pela ajuda, companhia e amizade. Sem dúvida, um bastião fundamental durante toda a minha estadia no Brasil.

Aos meus colegas Daniel, Gabrielle, Natalia, Mariana e Francisco pela ajuda na revisão deste trabalho.

Ao professor Sergio Fontoura, pelo interesse em orientar este trabalho e pela colaboração em realizar os ensaios de resistência e caracterização do conglomerado nos laboratórios da PUC-Rio.

Ao professor Alberto Sayão e ao professor Milton Kanji por aceitarem o convite para formar parte da banca avaliadora e por suas valiosas observações ao possibilitarem a melhora da qualidade do trabalho.

Ao geólogo Alexis Cerdas e ao engenheiro Eduardo Aviles pelas perguntas, dúvidas e comentários, que representaram a principal motivação para tentar abordar de forma sistemática o comportamento dos conglomerados.

Aos meus colegas, o engenheiro Esteban Vindas e geólogo Jorge Salazar, pela ajuda e comentários realizados na execução deste trabalho.

À equipe de engenharia do PHED pela colaboração na execução de trabalhos geotécnicos de campo: Mariana de los Ríos, Allan Rojas, Laura Villalobos, Cristian Delgado, Kelyn Rojas, Kevin Blanco, Roger Rodríguez, Manuel Guzmán, Heriberto Rojas, Roberto Guzmán, Errol Villanueva, Orlando Beita, Juan Fallas, Eder Rojas, Andrey Picado, Keneth Sibaja, Willfrido Fallas, Luis Solano, Baudilio Mena, Alvaro Carballo, Primo, Arcelio Arguedas.

Ao pessoal do CSD do ICE, em especial ao engenheiro Mauricio Varela e ao engenheiro Miguel Bolaños pela autorização para realizar este trabalho.

Aos técnicos do CSD do ICE pela execução dos ensaios geotécnicos de campo: Carlos Rosales, Alonso Araya, Luis Bermúdez e Walter Solano. 
Ao geólogo Manuel Barrantes do CSD do ICE pela ajuda nas análises petrografias.

À empresa Ingeotec S.A., especialmente ao engenheiro Marco Valverde, ao engenheiro Sergio Sáenz e ao técnico Carlos Ramirez pela ajuda na execução de ensaios granulométricos.

Ao engenheiro Victor Melendez, ao técnico Elizânio Ribeiro e ao técnico Rodrigo Alves pela execução dos ensaios de resistência e caracterização no LIRF-GTEP.

A Edson Silva pela ajuda na execução dos ensaios de caracterização no LGMA. 


\section{Resumo}

Jiménez Ugalde, Danilo Andrés; Fontoura, Sergio Augusto Barreto da (orientador). Caracterização e propriedades mecânicas de conglomerados. Rio de Janeiro, 2015. 139p. Dissertação de Mestrado Departamento de Engenharia Civil, Pontifícia Universidade Católica do Rio de Janeiro.

Os conglomerados são um tipo de rocha sedimentar, compostos pela mistura de blocos de rocha duros imersos em uma matriz fina branda. Os blocos no conglomerado fazem com que o estudo de suas propriedades mecânicas, através de ensaios convencionais de laboratório, seja de difícil execução. Por outro lado, os ensaios in-situ resultam mais representativos para definir as propriedades mecânicas deste tipo de materiais, mas em geral sua execução é muito demorada e representa um alto custo, o que justifica sua utilização somente em projetos de grande porte. Neste trabalho foi realizada a caracterização geotécnica de um conglomerado localizado no sul da Costa Rica, com o principal objetivo de definir, separadamente, as propriedades mecânicas dos blocos e da matriz. Os ensaios de caracterização e resistência utilizados nesta pesquisa foram realizados no laboratório de geotecnia do Instituto Costarriquense de Eletricidade e no laboratório de Interação Rocha-Fluido na PUC-Rio. Em uma segunda fase foi realizada a modelagem numérica do conglomerado através do método dos elementos finitos utilizando o programa PLAXIS 2D v2014. Da interpretação dos resultados dos ensaios numéricos foram obtidos os parâmetros de resistência e deformabilidade do conglomerado para diferentes escalas. Finalmente, os parâmetros mecânicos resultantes da modelagem numérica foram comparados com os obtidos na interpretação de ensaios de campo realizados na mesma litologia, especificamente ensaios de placa e cisalhamento direto multi-estagio, corroborando desta forma a validez da estimativa realizada.

\section{Palavras - chave}

Caracterização de conglomerados; ensaios de laboratório; método dos elementos finitos; bimrocks. 


\section{Abstract}

Jiménez Ugalde, Danilo Andrés; Fontoura, Sergio Augusto Barreto da (advisor). Characterization and mechanical properties of conglomerates. Rio de Janeiro, 2015. 139p. M.Sc. Dissertation - Departamento de Engenharia Civil, Pontifícia Universidade Católica do Rio de Janeiro.

Conglomerates are a type of sedimentary rock constituted by hard rock blocks embedded in a fine soft matrix. Due to the blocks present in the conglomerate, the study of mechanical properties by conventional laboratory tests is complicated. On the other hand, in-situ tests are more representative to define the mechanical properties of this type of materials; however, conducting these tests implies a lot of time and high costs, and their use is justified only for large-scale projects. This study provides the geotechnical characterization of a conglomerate located in the southern region of Costa Rica, where a large part of the works of the El Diquís Hydroelectric Project (PHED) will be built. The first objective of this research was to define the mechanical properties of the blocks and the matrix separately by using the results of characterization and strength tests. These tests were developed at the Geotechnical Laboratory of the Costa Rican Electricity Institute and the Rock-Fluid Interaction Laboratory at PUC-Rio. At a second research stage, numerical tests were conducted on virtual conglomerate samples. The creation of the virtual samples was based on the field and laboratory works performed during the first research stage. The numerical tests using virtual samples were carried out by applying the finite element method in the PLAXIS 2D 2014 program. The interpretation of the test results allowed determining the strength and deformability parameters of the conglomerate at different scales of the samples under analysis. Finally, the mechanical parameters derived from the numerical modeling were compared with the data from the interpretation of the field tests performed as part of the geotechnical studies at the dam site of the PHED, where plate load tests and multistage direct shear tests were carried out, thus corroborating the validity of the estimation that was made.

\section{Keywords}

Characterization of conglomerates; laboratoy tests; finite element method; bimrocks. 


\section{Sumário}

1. Introdução 24

1.1. Motivação 24

1.2. Objetivos do estudo 26

1.2.1. Objetivo geral 26

1.2.2. Objetivos específicos 26

1.3. Estrutura da dissertação 26

2. Revisão bibliografica $\quad 28$

2.1. Definição de conglomerado 28

2.2. Classificação dos conglomerados 29

2.2.1. Classificação geológica dos conglomerados 29

2.2.2. Classificação geomecânica da Sociedade Japonesa
de Geotecnia (JGS)

$\begin{array}{ll}\text { 2.2.3. Índice RMi } & 35\end{array}$

2.2.4. Índice de velocidade 36

2.3. Caracterização geotécnica dos conglomerados 37

$\begin{array}{ll}\text { 2.3.1. Resistência da matriz } & 37\end{array}$

2.3.2. Caracterização dos blocos 38

2.4. Modelagem de rochas clásticas de grão grosso 40

2.4.1. Modelagem física 40

$\begin{array}{ll}\text { 2.4.2. Modelagem numérica } & 41\end{array}$

2.4.3. Modelagem empírica: Bim strength criterion 45

2.5. Propriedades mecânicas dos conglomerados 49 
3. Metodologia utilizada na modelagem numérica dos conglomerados

3.1. Construção da amostra virtual do conglomerado 52

3.1.1. Definição do tipo de conglomerado 52

3.1.2. Definição da geometria externa da amostra 53

3.1.3. Construção do modelo mesoestrutural 54

3.1.4. Determinação dos parâmetros de resistência

e deformabilidade

55

3.2. Execução dos ensaios numéricos na amostra virtual 55

3.3. Interpretação dos ensaios numéricos 56

3.3.1. Ensaios de compressão uniaxial 56

3.3.2. Ensaios de compressão biaxial $\quad 57$

3.3.3. Ensaios de cisalhamento direto 58

3.3.4. Ensaios de placa $\quad 59$

4. Caso de estudo: Conglomerados meios e grossos da Fm. Paso Real, Costa Rica 61

$\begin{array}{ll}\text { 4.1. Importância do caso de estudo } & 61\end{array}$

4.2. Localização $\quad 62$

4.3. Contexto geológico 62

4.3.1. Geologia regional 62

4.3.2. Descrição geológica da Fm. Paso Real 70

4.3.3. Descrição litológica da Fm. Paso Real 71

4.4. Investigações geotécnicas 72

$\begin{array}{ll}\text { 4.4.1. Furos de sondagem } & 72\end{array}$

4.4.2. Galerias de investigação 73

$\begin{array}{ll}\text { 4.4.3. Afloramento } & 73\end{array}$

$\begin{array}{ll}\text { 4.4.4. Ensaios de laboratório } & 74\end{array}$ 
5. Apresentação e discussão dos resultados 78

5.1. Caracterização geotécnica do conglomerado 78

$\begin{array}{ll}\text { 5.1.1. Matriz } & 78\end{array}$

5.1.2. Blocos $\quad 89$

5.2. Classificação do conglomerado 95

5.2.1. Classificação geológica 95

5.2.2. Classificação segundo o sistema da JGS 95

5.2.3. Classificação segundo o sistema RMi 96

5.2.4. Classificação segundo o Índice de Velocidade 96

5.3. Modelagem numérica do conglomerado 97

5.3.1. Modelagem numérica da matriz do conglomerado
(escala de laboratório)

5.3.2. Modelagem numérica do conglomerado na escala de campo 106

6. Conclusões e recomendações para futuras pesquisas 124

$\begin{array}{ll}\text { 6.1. Conclusões } & 124\end{array}$

6.2. Recomendações para trabalhos futuros 127

$\begin{array}{ll}\text { Referências bibliográficas } & 128\end{array}$ 


\section{Lista de figuras}

Figura 2.1 - Exemplo de conglomerado: a) suportado pelos blocos, b) suportado pela matriz (Modificado de Tucker, 2001).

Figura 2.2 - Classes de arredondamento de grãos sedimentares. Para cada classe é apresentado um grão de baixa e outro de alta esfericidade (Modificado de Tucker, 2001).

Figura 2.3 - Sistema de classificação de maciços rochosos segundo suas propriedades fundamentais - extrato aplicável aos conglomerados (Modificado de Osada et al., 2005).

Figura 2.4 - Guia prático para a seleção do parâmetro $A$, dependente das propriedades de contato entre os blocos e a matriz (Kalender et al., 2014).

Figura 3.1 - Modelo conceitual utilizado na análise numérica dos conglomerados a) com matriz soldada, b) com matriz não soldada. Preto: blocos, Cinza escuro: matriz, Cinza claro: interface.

Figura 3.2 - Definição da geometria do modelo mesoestrutural: (a) Passo 1: Digitalização da amostra. (b) Passo 2: Modelo mesoestrutural formado pelos blocos e a matriz.

(c) Passo 3: Em amostras como matriz não soldada, inclusão da zona de interface entre os blocos e a matriz.

Figura 3.3 - Método do módulo de deformabilidade médio do trecho linear da curva tensão-deformação, utilizado no cálculo do módulo de deformabilidade nos ensaios de compressão uniaxial (Modificado de ISRM, 1979a).

Figura 3.4 - Envoltória de resistência no espaço das tensões principais, obtida dos ensaios de compressão (Modificado de ISRM, 1978). 
Figura 3.5 - Envoltória de resistência no espaço das tensões cisalhantes-tensões normais, obtida dos ensaios de cisalhamento direto (Modificado de ISRM, 1974).

Figura 4.1 - Localização geral do local de estudo, povoado de Buenos Aires, Puntarenas, Costa Rica.

Figura 4.2 - Mapa tectônico esquemático da região Mesoamericana com indicação das principais características tectônicas e movimentos relativos das placas: NR=Levantamento da Nicarágua; SE=Santa Elena; N=Nicarágua; HE=Escarpa de Hess; $\mathrm{CB}=$ Bacia da Colômbia; $\mathrm{PDB}=$ Cinturão deformado do Panamá; OF=Falha de Ocá; PF=Falha El Pilar; CRTFS=Sistema de Falha Transcorrente da Costa Rica; MAT=Trincheira Mesoamericana; CR=Dorsal de Cocos; PFZ=Zona de fratura de Panamá (Modificado de Astorga et al.,1991).

Figura 4.3 - Mapa tectônico esquemático da Costa Rica indicando as principais unidades morfotectônicas e bacias sedimentares (Modificado de Astroga et al., 1991).

Figura 4.4 - Coluna estratigráfica das bacias sedimentares da Costa Rica. A-D representam as quatro mega-sequências estratigráficas (Modificado de Bundschuh \& Alvarado, 2007).

Figura 4.5 - Exemplo de testemunhos de rocha recuperados dos furos de sondagem executados no conglomerado meio-grosso no local da barragem do PHED.

Figura 4.6 - Exemplo de galeria de investigação escavada no conglomerado meio-grosso no local da barragem do PHED (Cerdas et al., 2009).

Figura 4.7 - Afloramento de conglomerado meio-grosso investigado nesta pesquisa.

Figura 4.8 - Ensaios de campo: (a) Ensaio de cisalhamento direto. (b) Ensaio de placa. (Cerdas et al., 2009).

Figura 4.9 - Ensaio de cisalhamento direto multiestágio (Ensaios G2-4) realizado no conglomerado, no local da barragem do PHED.

Figura 5.1 - Distribuição granulométrica (em peso) das partículas menores de $32 \mathrm{~mm}$. (a) Local da barragem. (b) Local do afloramento. 
Figura 5.2 - Distribuição granulométrica (em volume) das partículas passando a peneira No. 40. (a) Local da barragem. (b) Local do afloramento.

Figura 5.3 - Petrografia realizada na matriz do conglomerado. (a) Amostra obtida no furo de sondagem PHB-RG-05. (b) e (d) Amostras obtidas no furo de sondagem PHB-RG-06. (c) Amostra obtida no furo de sondagem PHED-36-DSP.

Figura 5.4 - Relação entre o peso específico seco da matriz do conglomerado e: (a) Absorção. (b) Porosidade interconectada.

Figura 5.5 - Variação das propriedades da matriz do conglomerado com a profundidade: (a) Absorção. (b) Peso específico seco.

Figura 5.6 - Resultado dos ensaios de porosimetría de mercúrio: (a) Intrusão acumulativa de mercúrio. (b) Intrusão incremental de mercúrio.

Figura 5.7 - Módulo relativo da matriz do conglomerado segundo a classificação proposta por Ramamurthy (2004).

Figura 5.8 - Ensaio de compressão diametral (ensaio brasileiro) na matriz do conglomerado: (a) Corpo de prova antes da aplicação da carga. (b) Corpo de prova ao final do ensaio.

Figura 5.9 - Variação da resistência à tração em função da resistência à compressão uniaxial da matriz do conglomerado.

Figura 5.10 - Exemplo de envoltória do critério de ruptura de Mohr-Coulomb no espaço $\sigma_{1-\sigma} \sigma_{3}$ (Ensaio No. 1).

Figura 5.11 - Distribuição granulométrica (em peso) das partículas menores de $304,8 \mathrm{~mm}$ (12 polegadas) no local da barragem.

Figura 5.12 - Distribuição granulométrica das partículas no local do afloramento: (a) em área, e (b) em longitude. 
Figura 5.13 - Amostras dos diferentes tipos de rochas que formam os blocos do conglomerado: (a) andesito basáltico com textura fanerítica porfirítica, (b) andesito basáltico com textura fanerítica, (c) basalto andesítico com textura afanítica.

Figura 5.14 - Amostras virtuais para ensaios biaxiais na escala de laboratório (50×150 mm2): (a) amostra B1_50, (b) amostra B2_50, e (c) Amostra B3_50. Preto: blocos, Cinza: matriz, Azul: interface.

Figura 5.15 - Condições de contorno utilizadas nos testes biaxiais na escala de laboratório. Face superior: deslocamento horizontal nulo, deslocamento vertical uniforme, carregamento vertical uniforme. Face inferior: deslocamento nulo nos eixos horizontal e vertical. Faces laterais: deslocamento livre nos eixos horizontal e vertical, carregamento horizontal uniforme. As mesmas condições de contorno foram também aplicadas nos testes biaxiais na escala de campo.

Figura 5.16 - Ensaio de compressão biaxial ( $\left.\sigma_{3}=8 \mathrm{MPa}\right)$, executado na amostra B1_50, com os parâmetros de resistência do Caso 1: (a) primeiros pontos plásticos no corpo de prova $\left(\varepsilon_{a}=0,01\right)$, (b) ponto plásticos no final do trecho elástico $\left(\varepsilon_{a}=0,03\right)$, e (c) pontos plásticos no final do ensaio numérico $\left(\varepsilon_{a}=0,045\right)$. Branco: pontos plásticos por falha a tração. Vermelho: pontos plásticos por falha por cisalhamento.

Figura 5.17 - Ensaio de compressão uniaxial $\left(\left(\sigma_{3}=0 \mathrm{MPa}\right)\right.$ em amostras virtuais na escala de laboratório (Amostra B3_50, Caso 6): (a) amostra deformada no final do teste, (b) pontos plásticos no corpo de prova, e (c) deformação desviadora $\left(\gamma_{s}\right)$. Branco: pontos plásticos por falha a tração. Vermelho: pontos plásticos por falha por cisalhamento.

Figura 5.18 - Ensaio de compressão biaxial em amostras virtuais na escala de laboratório (Amostra B3_50, Caso 6, $\sigma_{3}=8 \mathrm{MPa}$ ): (a) amostra deformada no final do teste, (b) pontos plásticos no corpo de prova, e (c) deformação desviadora $\left(\gamma_{s}\right)$. Branco: pontos plásticos por falha a tração. Vermelho: pontos plásticos por falha de cisalhamento. 
Figura 5.19 - Resultado dos ensaios numéricos de compressão biaxial nas amostras virtuais na escala de laboratório, Amostra B2_50, Caso 2: (a) curvas tensão-deformação, e (b) envoltória de resistência bilinear no espectro das tensões confinantes analisadas.

Figura 5.20 - Resultados dos testes numéricos de compressão biaxial na escala de laboratório: (a) Resistência à compressão uniaxial, (b) coesão no intervalo das tensões $\sigma_{3}=0-2 \mathrm{MPa}$, (c) ângulo de atrito no intervalo das tensões de $\sigma_{3}=0-2 \mathrm{MPa}$, (d) coesão no intervalo das tensões $\sigma_{3}=2-8 \mathrm{MPa}$, (e) ângulo de atrito no intervalo das tensões de $\sigma_{3}=2-8 \mathrm{MPa}$.

Figura 5.21 - Resultados dos testes numéricos de compressão biaxial, realizados para estudar a deformabilidade das amostras virtuais da matriz do conglomerado.

Figura 5.22 - Amostras virtuais para ensaios biaxiais na escala de campo $(300 \times 900 \mathrm{~mm} 2)$ : (a) amostra B1_300, e (b) amostra B2_300.

Figura 5.23 - Amostras virtuais para ensaios biaxiais na escala de campo $(600 \times 1800 \mathrm{~mm} 2)$ : (a) amostra B1_600, e (b) amostra B2_600.

Figura 5.24 - Amostras virtuais para ensaios biaxiais na escala de campo $(900 \times 2700 \mathrm{~mm} 2)$ : (a) amostra B1_900, e (b) amostra B2_900.

Figura 5.25 - Resultado dos ensaios numéricos de compressão biaxial nas amostras virtuais na escala de campo, Amostra B2_600, Caso 3: (a) curva tensão-deformação, e (b) envoltória de resistência bilinear no espectro das tensões confinantes analisadas, típico para todos os testes biaxiais na escala de campo.

Figura 5.26 - Resistência à compressão uniaxial nas amostras virtuais na escala de campo. Preto: matriz. Branco: amostra horizontal. Cinza: amostra vertical.

Figura 5.27 - Coesão nas amostras virtuais na escala de campo, obtida através da interpretação dos ensaios de compressão biaxial: (a) intervalo das tensões de confinamento $\sigma_{3}=0-2 \mathrm{MPa}$, (b) intervalo das tensões de confinamento $\sigma_{3}=2-8 \mathrm{MPa}$. Preto: matriz. Branco: amostra horizontal. Cinza: amostra vertical. 
Figura 5.28 - Ângulo de atrito nas amostras virtuais na escala de campo, obtida através da interpretação dos ensaios de compressão biaxial: (a) intervalo das tensões de confinamento $\sigma_{3}=0-2 \mathrm{MPa}$, (b) intervalo das tensões de confinamento $\sigma_{3}=2-8 \mathrm{MPa}$. Preto: matriz. Branco: amostra horizontal. Cinza: amostra vertical.

Figura 5.29 - Resultados dos testes numéricos de compressão biaxial, realizados para estudar a deformabilidade das amostras virtuais do conglomerado na escala de campo.

Figura 5.30 - Amostras virtuais para ensaios de cisalhamento direto na escala de campo $(700 \times 700 \mathrm{~mm} 2)$ : (a) amostra C1_700, (b) amostra C2_700, (c) amostra C3_700, e (d) amostra C4_700. Preto: blocos, Cinza: matriz, Azul: interface.

Figura 5.31 - Condições de contorno utilizadas nos testes de cisalhamento direto na escala de campo. Face inferior: deslocamento nulo nos eixos horizontal e vertical. Face superior: deslocamento livre nos eixos horizontal e vertical, carregamento vertical uniforme. Lados inferiores: deslocamento horizontal nulo, deslocamento vertical livre. Lados superiores: deslocamento horizontal prescrito, deslocamento vertical livre.

Figura 5.32 - Ensaio de cisalhamento direto realizado na amostra C4_700, com os parâmetros de resistência do Caso 1 e uma tensão de confinamento $\sigma_{n}=8 \mathrm{MPa}$ : (a) primeiros pontos plásticos no corpo de prova, (b) pontos plásticos ao final do ensaio numérico, (c) amostra deformada ao final do teste, e (d) deformação desviadora $\left(\gamma_{s}\right)$. Branco: pontos plásticos por falha a tração. Vermelho: pontos plásticos por falha por cisalhamento.

Figura 5.33 - Ensaio numérico de cisalhamento direto realizados no conglomerado, amostra C2_700, Caso 2, $\sigma_{n}=2 \mathrm{MPa}$.

Figura 5.34 - Resultados dos ensaios numéricos de cisalhamento direto nas amostras virtuais na escala de campo, amostra C2_700, Caso 2, Caso 1: (a) curvas tensão-deslocamento, e (b) envoltória de resistência bilinear no espectro das tensões normais analisadas, típico para todos os ensaios cisalhantes na escala de campo. 
Figura 5.35 - Ensaio virtual de placa: (a) amostra virtual para ensaios de placa na escala de laboratório, (b) condições de contorno impostas na amostra virtual para os ensaios numéricos de placa: Face inferior: deslocamento nulo nos eixos horizontal e vertical. Lados: deslocamento horizontal nulo, deslocamento vertical livre. Face superior: deslocamento livre nos eixos horizontal e vertical, carregamento vertical uniforme de $45 \mathrm{~cm}$ de largura simulando a placa, (c) amostra virtual do conglomerado deformada ao final do ensaio numérico de placa (deslocamento $\times 20$ ). Preto: blocos, Cinza: matriz, Azul: interface. 


\section{Lista de tabelas}

Tabela 2.1 - Nomenclatura dos conglomerados (Richard, 2000).

Tabela 2.2 - Parâmetros de classificação e classes para maciços rochosos brandos (Osada et al., 2005).

Tabela 2.3 - Classes de grau de alteração do maciço rochoso (Osada et al., 2005).

Tabela 2.4 - Classificação da resistência do maciço rochoso segundo o índice RMi (Palmstrom, 1995).

Tabela 2.5 - Classificação da qualidade da rocha segundo o índice de velocidade (McDowell, 1993).

Tabela 2.6 - Três possíveis modos de propagação da ruptura observados em misturas de solo e rocha (Modificado de Xu et al., 2008)

Tabela 2.7 - Resumo de propriedades mecânicas dos conglomerados (modificado de Akram, 2010).

Tabela 4.1 - Ensaios de laboratório realizados no conglomerado.

Tabela 4.2 - Parâmetros de resistência do critério de ruptura de Mohr-Coulomb obtidos da interpretação dos ensaios de cisalhamento direto multiestágio in situ nos conglomerados, realizados no local da barragem do PHED (ICE, 2009a).

Tabela 5.1 - Distribuição granulométrica, em peso, das partículas menores de $32 \mathrm{~mm}$ da matriz do conglomerado.

Tabela 5.2 - Distribuição granulométrica, em volume, das partículas menores de $425 \mu \mathrm{m}$ (peneira No. 40) da matriz do conglomerado. 
Tabela 5.3 - Estatística descritiva do peso específico seco, absorção e porosidade interconectada da matriz do conglomerado.

Tabela 5.4 - Parâmetros obtidos dos ensaios de porosimetria por injeção de mercúrio realizados na matriz do conglomerado.

Tabela 5.5 - Estatística descritiva dos ensaios de propagação de velocidade de onda sônica realizados na matriz do conglomerado.

Tabela 5.6 - Estatística descritiva da resistência à compressão uniaxial, módulo de deformabilidade, e resistência à tração da matriz.

Tabela 5.7 - Classificação da rocha segundo sua resistência à compressão uniaxial (Ramamurthy, 2004).

Tabela 5.8 - Interpretação dos ensaios triaxiais utilizando o critério de ruptura de Mohr-Coulomb.

Tabela 5.9 - Estatística descritiva do peso específico seco, absorção e porosidade interconectada dos blocos do conglomerado.

Tabela 5.10 - Resultado dos ensaios de velocidade de onda realizados nos blocos do conglomerado.

Tabela 5.11 - Estatística descritiva da resistência à compressão uniaxial e módulo de deformabilidade dos blocos.

Tabela 5.12 - Estatística descritiva do índice da resistência da carga pontual e o valor do rebote do martelo Schmidt dos blocos do conglomerado.

Tabela 5.13 - Espessura da interface ao redor dos blocos na escala de campo.

Tabela 5.14 - Detalhe das amostras virtuais utilizadas nos ensaios biaxiais na escala de laboratório. 
Tabela 5.15 - Parâmetros de resistência dos materiais utilizados nos testes numéricos na escala de laboratório.

Tabela 5.16 - Parâmetros de deformabilidade e peso específico dos materiais utilizados nos testes numéricos realizado para estudar a resistência da matriz na escala de laboratório.

Tabela 5.17 - Módulo de deformabilidade, E (MPa) de cada material e resultado das análises realizadas na escala de laboratório.

Tabela 5.18 - Detalhe das amostras virtuais utilizadas nos ensaios de compressão biaxial na escala de campo.

Tabela 5.19 - Parâmetros de resistência e deformabilidade da matriz do conglomerado utilizados na análise da resistência na escala de campo.

109

Tabela 5.20 - Parâmetros de resistência e deformabilidade da interface do conglomerado utilizados na análise da resistência na escala de campo.

Tabela 5.21 - Parâmetros de resistência e deformabilidade dos blocos do conglomerado utilizados na análise da resistência na escala de campo.

Tabela 5.22 - Resumo dos parâmetros de resistência obtidos da interpretação dos ensaios numéricos biaxiais realizados nas amostras virtuais do conglomerado na escala de campo.

Tabela 5.23 - Comparação dos parâmetros de resistência obtidos dos testes numéricos de compressão biaxial na escala de campo com os valores calculados por meio de métodos empíricos (intervalo de tensões de confinamento $\sigma_{3}=0-2 \mathrm{MPa}$ ).

Tabela 5.24 - Módulo de deformabilidade, E (MPa) dos materiais utilizados nas análises do conglomerado na escala de campo.

Tabela 5.25 - Resultados dos testes numéricos de compressão biaxial, realizados para estudar a deformabilidade das amostras virtuais do conglomerado na escala de campo. 
Tabela 5.26 - Detalhe das amostras virtuais para ensaios de cisalhamento direto na escala de campo.

Tabela 5.27 - Resultados dos ensaios numéricos de cisalhamento direto realizado nas amostras virtuais do conglomerado.

Tabela 5.28 - Comparação dos parâmetros de resistência obtidos dos testes numéricos de compressão biaxial e dos ensaios numéricos de cisalhamento direto na escala de campo.

Tabela 5.29 - Comparação dos parâmetros de resistência obtidos dos ensaios de cisalhamento direto na escala de campo com os valores calculados por meio de métodos empíricos (intervalo de tensões normais $\sigma_{n}=0-1 \mathrm{MPa}$ ).

Tabela 5.30 - Resultados dos ensaios numéricos de placa realizados na amostra virtual do conglomerado.

Tabela 5.31 - Comparação do módulo de deformabilidade obtido dos ensaios numéricos de compressão biaxial e dos testes numéricos de placa, ambos na escala de campo. 


\section{Lista de símbolos}

$\begin{array}{ll}\text { ASTM } & \text { - American Society for Testing and Materials } \\ \text { Bimrock } & \text { - Block-in-matrix rock } \\ \text { E } & \text { - Módulo de deformabilidade } \\ \text { Fm. } & \text { - Formação geológica } \\ \phi & - \text { Ângulo de atrito } \\ \gamma & - \text { Peso específico } \\ \text { ISRM } & \text { - International Society for Rock Mechanics } \\ \text { JGS } & \text { - Japanese Geotechnical Society } \\ \text { MDF } & \text { - Método das diferenças finitas } \\ \text { MED } & - \text { Método dos elementos discretos } \\ \text { MEF } & - \text { Métodos dos elementos finitos } \\ \text { PVB } & - \text { Proporção volumétrica dos blocos } \\ \tau & - \text { Tensão cisalhante } \\ \text { UCS } & - \text { Resistência à compressão uniaxial } \\ \text { VER } & - \text { Volume elementar representativo } \\ \text { 2D } & - \text { Duas dimensões } \\ \text { 3D } & - \text { Três dimensões } \\ & \end{array}$

